\title{
On the Inverse Fourier Transform of the Planck-Einstein law
}

\author{
Alireza Jamali* \\ Senior Researcher \\ Natural Philosophy Department, Hermite Foundation ${ }^{\dagger}$ \\ alireza.jamali.mp@gmail.com
}

December 5, 2021

\begin{abstract}
After proposing a natural metric for the space in which particles spin which implements the principle of maximum frequency, $E=h f$ is generalised and its inverse Fourier transform is calculated. As a necessary result, a metric is found for the space in which quantum particles spin, hence the possibility of explanation of correlation of spacelike-separated particles is opened up.
\end{abstract}

Keywords - Einstein-Planck relation, foundations of quantum mechanics, principle of maximum frequency, angles-time space, renormalisation

There is a problem lurking at the foundations of quantum mechanics that is neglected and deserves much more attention: Taking Planck-Einstein relation $E=\hbar \omega$ as a fundamental law of nature and abiding by the principle that a fundamental law of nature must not depend on our choice of basis for the function space (Fourier basis), we expect the Inverse Fourier Transform of $E=\hbar \omega$ to yield the energy of electromagnetic field as a function of time, viz.

$$
E(t)=\frac{\hbar}{\sqrt{2 \pi}} \int_{-\infty}^{\infty} e^{i \omega t} \omega d \omega
$$

but this integral is divergent, and I maintain that this is the root of the problem of infinities (renormalisation) in Quantum Field Theory. It is not hard to see that this problem is inherited by quantum mechanics

\footnotetext{
${ }^{*}$ Corresponding author

†3rd Floor - Block No. 6 - Akbari Alley - After Dardasht Intersection - Janbazané Sharghi - Tehran - Iran
} 
and QFT as Schrödinger and Klein-Gordon equations are derived from the application of the operatorial form of Planck-Einstein-de Broglie law $p^{\mu}=\hbar k^{\mu}$ to the law of conservation of energy. Accordingly the resolution of this problem right at the beginning might help to overcome the problem of infinities. It is evident that if we are to get a proper inverse Fourier transform of $E=\hbar \omega$ we must correct it, and that is in fact what recent experiments suggest [1]. Mathematically however there are many ways to do so and without a firm physical motivation one can easily be led astray. To find such a firm physical ground we can approach the problem from two different perspectives which turn out to back up one another. One way is formally identical with the one followed in [2]: start from the

Principle. Maximum Angular Frequency ${ }^{1}$ All angular frequencies of rotation are bounded above by $\omega_{P}$ where

$$
\omega_{P}:=2 \pi f_{P}=2 \pi \sqrt{\frac{c^{5}}{\hbar G}} .
$$

This principle can be mathematically expressed by demanding the domain of $E(\omega)$ to be compact. The natural initial guess led by special relativity is the following

Definition. $\Omega$ factor

$$
\Omega:=\frac{1}{\sqrt{1-\left(\frac{\omega}{\omega_{P}}\right)^{2}}}
$$

which happens to satisfy all our expectations from a proper solution to the problem, by virtue of

- Respecting the Principle of Maximum Angular Frequency

- Having a smooth 'good' inverse Fourier transform

No other function can be found ${ }^{2}$ that satisfies these requirements simultaneously.

The second way to approach the problem is the following comparison

$$
E=\frac{1}{2} m \mathbf{v}^{2} \quad \text { versus, } \quad E=\frac{1}{2} I \boldsymbol{\omega}^{2}
$$

By analogy between $\mathbf{v}$ and $\boldsymbol{\omega}$, if there is a maximum for $\boldsymbol{v}$ then there must also be a maximum for $\boldsymbol{\omega}$, hence the principle of maximum angular frequency.

Contrary to the mainstream view that sees spin of elementary particles unrelated to any kind of rotational spin, I conjecture that it is the spin (rotational) motion of elementary particles that creates de Broglie matterwaves hence we must demand $E=\frac{1}{2} I \omega^{2}$ to yield $E=\hbar \omega$. To that end assuming that the spin (rotational) motion of an elementary particle is completely independent from its translational motion, we conclude

\footnotetext{
${ }^{1}$ As we also pointed in [2], this principle is in fact a sub-principle of the Principle of Ontologic Finiteness which will be stated in another paper, and justified to follow from the Epistemological Principle of Finiteness, which in turn can be considered a consequence of Descartes' notion of extension (res extensa) and Kant's a priori intuitions (Transzendentale $\ddot{A}$ sthetik).

${ }^{2}$ We have not still proved this statement, so this must remain at the level of a hypothesis. An investigation however is being done to prove it.
} 
that the spin motions must occur in an ontologically distinct space which we hereafter refer to as the space of angles-time. We postulate that the distances in this angles-time space are measured by the following metric

$$
d \Theta^{2}=\omega_{P}^{2} d t^{2}-d \boldsymbol{\theta}^{2}
$$

which naturally implements the principle of maximum frequency ${ }^{3}$. Another motivation for introducing a whole new independent space is to get close to explaining how particles which are separeted by space-like distances can still be correlated (quantum entanglement), for a new space with a new metric means that this space has a new independent notion of locality, independent of the locality in spacetime. As a result of the principle of maximum frequency the rotational energy of a particle is given by

$$
E=\frac{I \omega_{P}^{2}}{\sqrt{1-\left(\omega / \omega_{P}\right)^{2}}}
$$

By analogy with the gamma factor of special relativity we expect the $I \omega_{P}^{2}$ to yield the 'inertial' energy of rotation but that would only be the case if $I$ was a constant, but it turns out that if we are to recover $E=\hbar \omega$ from (3), $I$ is not a constant and depends on the frequency of rotation. To see this, observe that the second term in the Taylor expansions of (3) is

$$
\frac{1}{2} I \omega^{2}
$$

showing the expected compatibility with classical mechanics. But to get $E=\hbar \omega$ we need to use the definition of $I$ in terms of spin angular momentum $S$ and angular frequency $\omega$

$$
I=\frac{S}{\omega}
$$

which yields

$$
E=\frac{1}{2} S \omega
$$

which can be interpreted as meaning that wave-particle duality is a classical fact and it is in the quantisation of spin that quantum mechanics enters: to get $E=\hbar \omega$ we now only need to let

$$
S=2 \hbar
$$

in (4). This $S=2 \hbar$ is nothing but an 'old' quantum-mechanical quantisation rule. Putting all these considerations together, complete exact form of Planck-Einstein relation is given by

$$
E(\omega)=\frac{2 \hbar \omega_{P}^{2}}{\omega}\left(\frac{1}{\sqrt{1-\left(\omega / \omega_{P}\right)^{2}}}-1\right)
$$

\footnotetext{
${ }^{3}$ This metric has many other benefits, in particular yielding a natural satisfactory kinematical definition for the spin vector of a particle. These topics are explored in [3], which is the precursor of this work.
} 
Note that by construction $E=\hbar \omega$ is an approximation to the above equation, by being the first term in the Taylor expansion of the anglestime space factor. Unlike $E=\hbar \omega$ however, our proposed generalisation of Planck-Einstein relation is not plagued by infinities and it is perfectly possible to take its inverse Fourier transform. To this purpose, recall that

$$
\mathcal{F}_{\omega}^{-1}\left[\frac{1}{\sqrt{1-\left(\omega / \omega_{P}\right)^{2}}}\right]=-i \omega_{P} \sqrt{\frac{2}{\pi}} K_{0}\left(-i \omega_{P} t\right),
$$

where $K_{0}(t)$ is the zeroth order modified Bessel function of the second kind. Now observe that

$$
i \omega E(\omega)=2 i \hbar \omega_{P}^{2}\left(\frac{1}{\sqrt{1-\left(\omega / \omega_{P}\right)^{2}}}-1\right),
$$

which is

$$
\mathcal{F}\left[\frac{d}{d t} E(t)\right]=-2 i \hbar \omega_{P}^{2} \mathcal{F}\left[i \omega_{P} \sqrt{\frac{2}{\pi}} K_{0}\left(-i \omega_{P} t\right)+\delta(t)\right],
$$

therefore

$$
E(t)=-2 i \hbar \omega_{P}^{2}\left(1+i \omega_{P} \sqrt{\frac{2}{\pi}} \int_{0}^{t} K_{0}\left(-i \omega_{P} \tau\right) d \tau\right)
$$

\section{References}

[1] Dakotah Thompson et al. Hundred-fold enhancement in far-field radiative heat transfer over the blackbody limit. Nature, 561:216-221, 2018.

[2] Alireza Jamali. Relativity of energy. 10.20944/preprints202111.0559.v1, 2021.

[3] Alireza Jamali. Towards an einsteinian quantum mechanics. viXra:2103.0006, 2021. 